\title{
ORIGINAL
}

\section{BROTE DE SÍNDROME DE SHOCK TÓXICO ESTREPTOCÓCICO EN UNA GUARDERÍA DE CANTABRIA EN 2006}

\author{
Manuel Ortega-Mendi (1), Luís Martínez-Martínez (2), Alvaro González de Aledo-Linos (1), \\ Jesús Agüero-Balbín (2), Luís Viloria-Raymundo (1), Mª Eliecer Cano- García (2), Jorge Calvo- \\ Montes (2), Purificación Mellado-Encinas (3) y Amparo Fernández-Rodríguez (4). \\ (1) Servicio de Salud Pública. Dirección General de Salud Pública. Consejería de Sanidad del Gobierno de Cantabria. \\ (2) Servicio de Microbiología. Hospital Universitario Marqués de Valdecilla. Santander. \\ (3) Sección de Microbiología. Hospital de Laredo. Cantabria. \\ (4) Laboratorio de Microbiología. Servicio de Biología. Instituto Nacional de Toxicología y Ciencias Forenses. Madrid.
}

\section{RESUMEN}

Fundamento: Las infecciones por estreptococo betahemolítico grupo A (EGA) sólo excepcionalmente son agresivas y con letalidad alta. Más infrecuente aún es la ocurrencia de un brote. El objetivo de este estudio es la descrpción de un brote epidémico por estreptococo beta-hemolítico grupo A en una guardería de Cantabria.

Métodos: Estudio descriptivo de un brote de síndrome de shock tóxico estreptocócico ( 3 casos, uno letal) en una guardería, que motivó una intervención de salud pública con quimioprofilaxis, cierre de la guardería y estudio de los contactos. Se analizan los determinantes de la infección en los casos invasivos y no invasivos, y los resultados de los cultivos faríngeos de los contactos.

Resultados: Se identificaron 3 casos invasivos y 14 no invasivos entre los 40 niños de la guardería (tasa de ataque $42,5 \%$ ). Se estudiaron 19 posibles determinantes de la infección, asociándose sólo la edad mayor de 24 meses y la asistencia al aula de fichas (la de los niños más mayores). No se asoció a la varicela. Se investigaron microbiológicamente todos los niños de la guardería y su personal (4 cuidadoras) y 258 personas de contacto. En 12 de los niños se aisló el estreptococo eтm 4, incluyendo 2 de los 3 casos con enfermedad invasiva. En 13 de los 258 contactos se aislaron otras cepas de estreptococo, pero en ninguno la causante del brote. Se hizo quimioprofilaxis con azitromicina a todos los niños y contactos, y a los positivos se les repitió el tratamiento hasta su negativización.

Conclusiones: La cepa invasiva circuló sólo en la guardería. La quimioprofilaxis erradicó efectivamente la infección.

Palabras clave: Estreptococo piógenes. Síndrome de shock tóxico. Guardería.

Correspondencia:

Manuel Ortega Mendi.

Federico Vial, 13

39009 Santander

Correo electrónico: ortega_m@gobcantabria.es

\section{ABSTRACT}

\section{Outbreak of Streptococcal Toxic Shock Syndrome in a Day Care Center in Cantabria, Spain, 2006}

Background: Beta hemolytic group A streptococcus only exceptionally produces aggressive disease with high lethality. Even more uncommon is the occurrence of an outbreak. In Spain, no outbreak in child care center has been previously described.

Methods: Descriptive study of an outbreak of streptococcal toxic shock syndrome ( 3 cases, one lethal) in a child care center, which motivated the health care intervention with chemoprophylaxis, the closure of the child care center and the study of contacts. We analyzed the determinants of infection in the invasive and non-invasive cases, and the results of the pharyngeal culture of contacts.

Results: We identified 3 invasive and 14 non-invasive cases between 40 children attending the child care center (attack rate $42.5 \%$ ). We studied 19 possible determinants of the infection, finding only an association with being over the age of 24 months and the assistance to the handouts classroom (that of the oldest children). It was not associated with chickenpox. All children attending the child care center, its staff (4 women) and 258 contacts were microbiologically investigated. In 12 children the emm 4 strain was isolated, including 2 of 3 cases with invasive disease. In 13 of 258 contacts other strains of beta hemolytic group A streptococcus were isolated, but in none of them the strain responsible of the outbreak was found. Azytromicin chemoprophylaxis was implemented for all children and contacts, and in those with a positive isolation, the culture was repeated until negative.

Conclusions: The invasive strain circulated only in the child care center. Azytromicin chemoprophylaxis eradicated effectively the infection.

Key words: Streptococcus Pyogenes. Toxic Shock Syndrome. Child daycare center. 


\section{INTRODUCCIÓN}

El Estreptococo Beta-hemolítico del Grupo A (EGA) está presente con frecuencia en la garganta o la piel de los humanos, de forma asintomática o provocando enfermedades leves (amigdalitis, escarlatina o impétigo). Pero en ocasiones origina enfermedades graves como la fascitis necrotizante o el síndrome de shock tóxico estreptocócico (SSTE), éste último con una letalidad de hasta el $60 \%{ }^{1-3}$. La diferente evolución clínica se atribuye al estado inmunitario del huésped y/o las características del microorganismo. En 2002 microbiólogos de 11 países europeos iniciaron el Strep-EURO, un proyecto de investigación y vigilancia de enfermedad invasiva (EI) por EGA para conocer su epidemiología en nuestro continente. En Europa la incidencia de EI varía entre 0,4-4,8/100.000/año y en EEUU es de $3,5^{4,5}$. España no participa en el StrepEURO y la EI por EGA no es de declaración obligatoria, por lo que se desconoce su incidencia. En 2005 se declararon 28 casos al Sistema de Información Microbiológica (SIM), pero al ser la declaración voluntaria esta información no es adecuada para calcular incidencia. Los brotes son menos frecuentes: en España solo hay descripciones en personas drogadictas ${ }^{6}$ y en Cantabria no existe ningún antecedente. De momento no hay protocolos para la EI en nuestro país.

Este trabajo describe el primer brote de SSTE en una guardería descrito en España, que afectó a 3 niños, identificando los factores de riesgo y evaluando las medidas de control tomadas.

\section{SUJETOS Y MÉTODOS}

Descripción del brote. El 31-3-2006 una niña de 32 meses (caso 1) falleció tras un fallo multiorgánico que había debutado el día anterior. Ese mismo día ingresó un niño de 39 meses (caso 2) que acudía a la misma guardería de Castro Urdiales, Canta- bria, con coma y fallo multiorgánico. El 4 de abril un tercer niño (caso 3 ) de 15 meses de la misma guardería ingresó por shock severo y fallo multiorgánico. Los dos últimos se recuperaron con secuelas leves. Antes de disponerse de resultados microbiológicos, los dos primeros se interpretaron como enfermedad meningocócica, realizándose quimioprofilaxis el 31-3-06 a los 38 restantes compañeros de guardería con rifampicina, y a las 4 cuidadoras con ciprofloxacino.

El 4 de abril la necropsia del caso 1 atribuyó su muerte al SSTE. Ante este diagnóstico y el ingreso del tercer caso, el 5-4-06 se realizó una segunda quimioprofilaxis con azitromicina a todos los niños, cuidadoras y convivientes directos. La pauta consistió en $12 \mathrm{mg} / \mathrm{K} /$ día (máximo $500 \mathrm{mg}$ /día) en una sola toma diaria, durante 5 días, y se aplicó a 258 personas. Esta pauta se eligió entre las 3 que recomienda el CDC por su eficacia (95\% de erradicación), su comodidad al ser por vía oral, su corta duración, y porque nos constaba la sensibilidad del germen a los macrólidos ${ }^{7}$.

El 3-4-2006 técnicos de Salud Pública inspeccionaron la guardería para estimar la situación de higiene e identificar vehículos de transmisión, sin encontrar ninguna irregularidad. En la guardería no se servían comidas y no se recogieron muestras ambientales. A pesar de ello, para limitar los contactos mientras hiciera efecto la quimioprofilaxis, se ordenó su cierre del 6 al 12 de abril, con una limpieza a fondo antes de la reapertura. A mediados de mayo se ordenó retirar de todas las guarderías de Cantabria, públicas y privadas, los juguetes que imitan productos alimenticios, cubiertos o teléfonos, por su mayor riesgo de transmisión de gérmenes al facilitar conductas de riesgo.

Durante el brote otros niños de la guardería y convivientes presentaron síntomas como fiebre y tos. Siete niños de la guarde- 
ría y 3 niños convivientes fueron ingresados para observación, descartándose la infección estreptocócica.

Definiciones de caso y contactos. Los casos se buscaron entre quienes consultaron al médico en Castro Urdiales con síntomas de infección, teniendo relación con la guardería o contacto con alguien que acudiera a la guardería, tras pedir a los médicos que extremaran la vigilancia y los declararan. Se aplicaron las siguientes definiciones: Caso con SSTE: clínica compatible y cultivo positivo para EGA, o clínica compatible sin otra causa microbiológica y vínculo epidemiológico con los anteriores. Caso con cultivo: niño de la guardería que tuviese un cultivo positivo para EGA entre febrero y abril 2006. Caso con Antiestreptolisina O (ASLO): niño de la guardería con ASLO positivo (> $200 \mathrm{UI} / \mathrm{ml}$ por turbidimetría) entre febrero y abril 2006.

Para el análisis se agruparon los casos de esas tres categorías en: Caso con EGA o portador: niño de la guardería con cultivo positivo, detección de ASLO, o SSTE entre febrero y abril de 2006. Contacto investigado: persona relacionada con la guardería a la que se realizó frotis y tratamiento. Contacto conviviente: quien hubiera compartido hogar durante al menos 4 horas al día, cinco días a la semana, en las últimas 2 semanas; incluyeron padre/madre, hermanos, tíos, amigos, abuelos, familia cuidadora y "otros".

Epidemiología descriptiva. Se estudiaron la edad, sexo, y grupo en la guardería; se consultaron las historias desde febrero de 2006, la incidencia de síntomas, las veces que acudieron al médico y las fechas de ingreso. Se construyeron variables bimodales de edad, horas de estancia, etc., y se calcularon las tasas de ataque por grupos según presentación de síntomas y factores determinantes.

Epidemiología analítica. Se realizó un estudio de cohorte retrospectiva en la guar- dería a partir de las historias clínicas y la información recogida en la guardería. La población de estudio fueron los niños de la guardería desde el 1-2-06 hasta el 25-4-06. Se utilizó el paquete de software EPI-dat 3.1 para determinar riesgos relativos (RR) e intervalos de confianza (95\%) para los posibles factores determinantes.

\section{Estudio microbiológico}

Se realizaron cultivos de los frotis faríngeos pertenecientes a todos los asistentes a la guardería, incluyendo niños, cuidadoras y otro tipo de personal del centro, así como de los contactos convivientes. Se estudiaron también otro tipo de muestras invasivas pertenecientes al caso 1.

El estudio microbiológico y la caracterización molecular de los aislamientos se llevó a cabo en los siguientes centros: Instituto Nacional de Toxicología y Ciencias Forenses, Servicio de Microbiología del Hospital Universitario Marqués de Valdecilla, Centro Nacional de Microbiología del Instituto Carlos III y Laboratorio de Microbiología del Hospital de Laredo.

La caracterización de los aislamientos de los cultivos incluyó: tipificación por secuenciación del gen emm que codifica la proteína M tipoespecífica; relación clonal, por electroforesis en campo pulsado (PFGE); determinación de factores de virulencia, por detección de genes codificadores de toxinas pirogénicas; y sensibilidad antibiótica, por estudios de CMI a distintos antimicrobianos (J. Agüero et al 2007. remitido para publicación).

\section{RESULTADOS}

Epidemiología descriptiva. La guardería disponía de tres aulas: de cunas (para los más pequeños, $\mathrm{n}=10$ ), multiusos (para los que ya andan, $n=10$ ) y de fichas (para los 
más activos, $\mathrm{n}=20$ ). Acudían por tanto 40 niños, así como 4 cuidadoras. Los niños tenían entre 5 y 40 meses (mediana 29), el $57,5 \%$ eran niñas, y acudían entre 2,5 y 8,5 horas al día (mediana 4 horas). Entre los 40 niños se detectaron 17 casos con EGA o SSTE $(42,5 \%)$ todos mayores de 12 meses (Tabla 1). Trece tenían cultivos positivos, tres ASLO, y uno era negativo pero con vínculo epidemiológico y SSTE. Se identificaron tres niños con SSTE (tasa de ataque $7,5 \%$ ) dos confirmados por cultivo y uno por vínculo epidemiológico:

Caso 1 (por cultivo): niña de 32 meses, melliza, pretérmino de 28 semanas con historia de distress respiratorio grave del recién nacido, sepsis, hemorragia intraventricular y bronquiolitis. Cuatro semanas antes tuvo neumonía y 18 días antes varicela. Consultó el 30-3-06 por fiebre, vómitos y dolor abdominal y falleció el 31 por fallo multiorgánico. Caso 2 (por vínculo epidemiológico): niño de 39 meses que ingresó el 31-3-06 por shock, fallo multiorgánico, insuficiencia renal, rabdomiolisis, trombosis safena, encefalopatía y coma, que se recuperó sin secuelas tras un ingreso prolongado. Caso 3 (por cultivo): niño de 15 meses que ingresó el 4-4-06 con shock severo, fallo multiorgánico y necrosis isquémicas distales, que se recuperó tras un ingreso prolongado con defectos cicatriciales en los dedos. Los casos 2 y 3 tenían antecedentes inespecíficos de patologías intercurrentes. De los otros 14 casos con EGA, 9 acudieron al médico con síntomas respiratorios y 5 permanecieron asintomáticos.

Se revisaron las historias de 33 niños de la guardería (los restantes carecían de historia en el Centro de Salud) desde el 01-0206, identificándose 75 consultas. La mayoría fueron procesos inespecíficos, mereciendo destacar 4 casos de varicela (incluyendo el caso 1, el único positivo posteriormente para EGA entre los cuatro con varicela).
Tabla 1

Distribución de todos los niños de la guardería $(n=40)$ por numero de casos, edad, sexo y aula, y distribución de los "casos" de la guardería $(n=17)$

\begin{tabular}{|l|c|c|c|c|}
\hline & $\begin{array}{c}\mathbf{N}^{\mathbf{0}} \\
\text { de niños }\end{array}$ & $\begin{array}{c}\% \\
\text { de niños }\end{array}$ & $\begin{array}{c}\mathbf{N}^{\mathbf{0}} \\
\text { de casos }\end{array}$ & $\begin{array}{c}\% \\
\text { de casos }\end{array}$ \\
\hline Edad (meses) & & & & \\
$0-12$ & 6 & 15,0 & 0 & 0 \\
$13-24$ & 11 & 27,5 & 3 & 18 \\
$25-36$ & 16 & 40,0 & 8 & 47 \\
37 o más & 7 & 17,5 & 6 & 35 \\
\hline Sexo & & & & \\
Mujer & 24 & 60 & 6 & 35 \\
Varón & 16 & 40 & 11 & 65 \\
\hline Aula & & & & \\
Clase fichas & 20 & 50 & 12 & 71 \\
Cuna & 10 & 25 & 2 & 12 \\
Multiusos & 10 & 25 & 3 & 18 \\
\hline
\end{tabular}

Se investigaron 258 personas de contacto (239 con los niños, 19 con las cuidadoras), lo que representa seis contactos por niño (rango: 0-13). De estas 258 personas 13 fueron positivas $(5 \%)$, mayoritariamente hermanos (15,4\% de positivos) y padres $(7,6 \%)$. No hubo positivos entre personal de la guardería, cuidadores u otros familiares. A todas las personas investigadas se les trató con azitromicina, y en los positivos se repitió el tratamiento hasta su negativización. En cinco se encontró estreptococo del grupo $\mathrm{C}$.

Respecto a los contactos de los casos de SSTE, del caso 1 se investigaron tres (hermano mellizo y padres) que resultaron negativos; del caso 2 se investigaron 12 (todos negativos); y del caso 3 se investigaron 10 (uno positivo, de distinta cepa).

Epidemiología analítica. Las tasa de ataque fue de $61 \%$ en niños $>2$ años y de $18 \%$ en $<2$ años (RR 3,5; IC 95\%: 1,2$10,1)$. Por aulas fue del $20 \%$ en el de cunas, $30 \%$ en el de multiusos y $60 \%$ en el de fichas. Se investigaron 19 posibles determinantes de la infección (de cualquier gravedad): asistir $>4$ horas/día a la guardería o desde hace $>6$ meses, estar en el aula de fichas, haber tenido $>3$ enfermedades 0 varicela desde febrero, tener algún familiar con EGA, edad > 24 meses, exantema, conjuntivitis, fiebre, hiperemia faríngea, rino- 
rrea, catarro, tos, dolor abdominal, diarrea, vómitos, uso de antibióticos, o uso de antiinflamatorios no esteroideos. Sólo se asociaron a mayor riesgo la edad $>24$ meses (RR 3,45, IC 95\%: 1,17-10,14) y el aula de fichas (RR 2,40, IC95\%: 1,04$5,55)$. En este brote la varicela no se asoció a infección por EGA de cualquier gravedad (RR 0,77, IC95\%: 0,15-3,98), ni el uso previo de antiinflamatorios no esteroideos (RR 1,30, IC95\%: 0,64-2,66). Se estudió la posible asociación de la varicela con la forma grave de la infección (SSTE) encontrándose un RR 4,11 (IC 95\%: 0,29-28,42), no significativo.

Microbiología. Se estudiaron 39 aislados de EGA procedentes: cinco del caso 1; un hemocultivo del caso 3; 10 faríngeos de niños de la guardería; trece faríngeos de los contactos; un faríngeo de una mujer de Santurce no relacionada con el brote; y nueve de la colección de microorganismos del Hospital Valdecilla, de pacientes no relacionados con el brote.

Los estreptococos de los casos 1 y 3 estuvieron relacionados clonalmente con los de los demás niños de la guardería [mismos patrones de PFGE, mismo tipo emm 4, y mismo perfil de genes productores de toxinas (exotoxinas pirogénicas SpeB, SpeC, SpeF, SmeZ y SSA)]. Esta cepa de la guardería fue diferente de las cepas de los contactos y de las otras cepas aisladas en Cantabria ajenas al brote, tanto en el patrón de PFGE como en el tipo emm y el perfil de producción de toxinas. Un solo caso no relacionado portaba la misma cepa de la guardería: la madre de un niño de Santurce que había ingresado por eritrodermia y fiebre, con cultivos negativos. Ella había presentado la semana anterior un cuadro semejante que cedió sin tratamiento, y se encontró la misma cepa de la guardería en su faringe. Era profesora de un colegio a 360 metros de la guardería, pero no se pudo encontrar ningún vínculo epidemiológico con el brote.

\section{DISCUSIÓN}

En la guardería hubo tres casos de SSTE, lo que teniendo en cuenta su baja incidencia se puede considerar un brote. Aunque la mayoría de los casos publicados son aislados, hay descripciones de clusters en familias $^{8,9}$, cuarteles ${ }^{10}$, residencias de ancia$\operatorname{nos}^{11}$, equipos deportivos ${ }^{12}$, escuelas $\mathrm{y}$ guarderías ${ }^{1,13}$. Se cree que la incidencia de EI por EGA en Europa está creciendo desde los años $80^{4}$. Un estudio de Valencia en 2005 mostró una tendencia ascendente en cinco años ${ }^{14}$, pero los datos del SIM no muestran dicha tendencia. El brote de Castro Urdiales no parece ser consecuencia de un aumento general de la enfermedad en España.

En nuestros 3 casos no encontramos enfermedad subyacente. Estudios previos identificaron como factores de riesgo en niños: tener un padre que fuma o más de un niño mayor en casa, el antecedente de varicela, o iniciar el consumo de antiinflamatorios no esteroideos, y en adultos: edad $\geq 65$ años, infección VIH, diabetes, cáncer, cardiopatía, uso de drogas IV, abuso de alcohol o tratamiento con esteroides $2,5,7,13,15,16$. Por el contrario como factores de protección: el mayor número de habitaciones en casa, un mayor nivel educativo de los padres, y tener un familiar con rinitis en las 2 últimas semanas -la infección respiratoria con rinitis indica viriasis, mientras que sin rinitis es más probablemente estreptocócica- ${ }^{15}$. En el estudio de Valencia todos presentaron alguna enfermedad subyacente ${ }^{14}$. Nuestro caso 1 podría haber estado relacionado con la varicela 18 días antes, pues la varicela aumenta 58 veces el riesgo de EI y 5 veces el de muerte, y entre el 15 y el $25 \%$ de los casos pediátricos de EI tienen el antecedente de varicela ${ }^{7,15-18}$. En la semana del 20-26 de febrero Castro Urdiales tenía una tasa de varicela 3,4 veces más alta que el promedio de Cantabria y en la guardería hubo otros 3 niños con varicela en los últimos 2 meses, pero no existió asociación estadísticamente 
significativa con el SSTE, aunque el número tan pequeño de casos impide descartar la asociación. También se investigó el uso previo de antiinflamatorios no esteroideos, no comprobándose asociación ${ }^{19}$. Como en las historias clínicas no figuraban otros factores como número de personas y hermanos en la casa, tabaquismo, condiciones de la vivienda, etc., no se pudieron investigar otros determinantes.

Se identificó EGA en el $42,5 \%$ de los niños de la guardería, y de ellos el $100 \%$ con la misma cepa, que no se encontró en convivientes ni en la población general. Esto sugiere que la transmisión ocurrió en la guardería, tal vez por infecciones respiratorias antes del brote, hacinamiento, o por fómites. Este último mecanismo se sospechó en una residencia de ancianos ${ }^{11} \mathrm{y}$ en una guardería en que se aisló la cepa responsable en 6 de 112 muestras ambientales (todas eran juguetes de plástico imitando comidas, lo que posiblemente facilitó la transmisión oral $)^{13}$. En nuestro caso no se pudo demostrar al no tomar muestras ambientales. Como comparación, en una escuela de Minnesota estudiada en el contexto de un brote, el $78 \%$ de los niños portadores lo eran de la cepa invasiva causante de los casos, frente al $100 \%$ en Castro Urdiales $^{1}$. En la guardería no había animales de compañía (perros o gatos) que se han descrito como posibles reservorios y causantes de brotes de repetición ${ }^{20}$.

Se desconoce la prevalencia de la infección en Cantabria. En niños asintomáticos menores de 3-4 años en guarderías de EEUU y Suecia se han descrito prevalencias de 1,3 y $3,4 \%$ respectivamente ${ }^{21}$. En Pensilvania se investigó un grupo de 5-15 años de edad durante 4 años, resultando positivos el $16 \%{ }^{22}$. En el contexto de brotes, en una guardería en Boston el 35\% de los niños de 3-4 años de la misma clase eran portadores, y el $8 \%$ de otras clases ${ }^{13}$, en una escuela/guardería de Taiwán el 5,6\% , y en una guardería de EEUU el $36 \%{ }^{21}$. La mayor prevalencia en nuestro brote $(42,5 \%)$ puede incluso estar infraestimada por la intervención previa con rifampicina/ciprofloxacino. Todos los aislados de la guardería fueron muy sensibles a antimicrobianos (salvo dos resistentes a rifampicina que se atribuyen a su administración previa) y coincidieron con los aislados de la niña fallecida. Las cepas de los contactos también fueron sensibles.

El cultivo es la forma mas adecuada de diagnosticar EGA, con sensibilidad de $99.5 \%$ y especificidad de $100 \%{ }^{23}$. En este brote todos se tomaron de orofaringe. En trabajadores sanitarios relacionados con brotes el sitio más habitual de encontrar el EGA fue el ano, así como la vagina, la piel y la faringe ${ }^{24}$. En brotes de escarlatina en guarderías también se ha sospechado la existencia de portadores no faríngeos y el mecanismo de transmisión distinto de las gotitas respiratorias ${ }^{8}$. Desconocemos la prevalencia de portadores no faríngeos en la población general, así como en este brote, al no tomarse frotis de esas localizaciones.

El brote de Castro Urdiales coincide con las estaciones de máxima incidencia (invierno y primavera) ${ }^{25,26}$ pero se desconoce si es simplemente por el mayor número de enfermedades respiratorias en ellas. El EGA se transmite por gotitas respiratorias, y las personas sintomáticas son probablemente más infecciosas que las asintomáticas; además un EGA que ha causado EI es más probable que vuelva a causarla en sucesivos $\operatorname{contagios}^{16}$. Los estreptococos que infectan la faringe podrían regular la expresión de los genes que median la virulencia y la evasión de la defensa inmunológica, y así transformarse en un tipo más invasivo para otras personas $^{27,28}$. Tal vez ocurriera esto en la guardería, pues la mayoría de los niños con EGA presentaba síntomas respiratorios. Pero la hipótesis alternativa es que la susceptibilidad tenga un componente perso- 
nal, ligado a variaciones de cada individuo, lo que explicaría que la misma cepa se aislase de 13 niños pero sólo tres desarrollaran el SSTE.

Es razonable pensar que la cepa llegó a la guardería a través de alguno de los niños, pero no necesariamente los que desarrollaron el SSTE. El riesgo de SSTE para los contactos fuera de la guardería parece bajo, ya que no portaban el tipo causante del brote; a la luz de este dato su quimioprofilaxis parece innecesaria, pero en el momento de darla se desconocía. Aunque el riesgo de EI en contactos íntimos es más alto que en la población general, en términos absolutos es bajo (66-294/100.000) y se concentra en el mes siguiente al inicio de la enfermedad en el caso índice, aunque mayoritariamente en los 7 días siguientes $^{5,18,29}$. La azitromicina puede eliminar el EGA de los portadores ${ }^{7}$ pero no hay datos sobre la efectividad de ninguna pauta para prevenir la EI, que se deduce racionalmente de la erradicación de la faringe ${ }^{7,18,29}$. Así, en el consenso del CDC de los Estados Unidos y en el protocolo del Health Protection Agency (HPA) del Reino Unido 5,7,30,31 no se recomiendan frotis ni quimioprofilaxis en los contactos íntimos, aunque el CDC la admite para aquellos con factores de riesgo $^{7}$. El protocolo más reciente de Canadá de octubre de 2006 la indica para los contactos íntimos de los casos con EI, definidos como 4 horas al día en los 7 días previos $^{18}$.

Respecto a las guarderías, el CDC las excluye de forma explicita de la definición de "contactos íntimos" pero no da recomendaciones para ellas ${ }^{7}$. El protocolo de Canadá ${ }^{18}$ recomienda que ante un caso de EI se dé quimioprofilaxis en la familia y los "centros de cuidado de niños en domicilio" (forma de cuidado infantil excepcional en España); en guarderías "institucionales" sólo si hay más de un caso de EI en un mes o si coexiste una epidemia de varicela. En el brote en Castro Urdiales los contactos entre los niños parecen al menos tan íntimos como los domiciliarios, pues aunque sólo asistieran un promedio de cuatro horas al día durante un máximo de cinco días a la semana, la transmisión se produjo. Si se consideran los contactos en una guardería española parecidos a los "contactos íntimos" podemos afirmar que cuando aparece más de un caso de EI dentro de 30 días, estaría indicada la profilaxis a todos los niños, y de acuerdo con varias fuentes, incluso ante un solo caso. Además si los casos secundarios aparecen en poco tiempo se justifica la profilaxis sin esperar al resultado de los cultivos ${ }^{18}$. Así lo hicimos en Cantabria el mismo día en que se conoció el diagnóstico. Respecto al cierre de la guardería, no hemos encontrado antecedentes en la literatura y pudo haber tenido un efecto perverso: algunos padres solicitaron ingresar a sus hijos en otra guardería, con el riesgo de introducir el germen en otro colectivo. Pare evitarlo se ordenó que antes de volver a la guardería del brote, o de ingresar en cualquier otra, se exigiese un certificado de frotis negativo emitido por su pediatra.

Tras la experiencia de este brote se pueden hacer la siguientes recomendaciones: ante un caso de EI en una guardería, investigar a los otros niños y tratar sólo a los que tengan síntomas o cultivo positivo (este es el punto más polémico y podemos sentirnos presionados para tratar a todos); ante más de un caso, dar profilaxis a todos los niños sin esperar los resultados; no dar sistemáticamente profilaxis fuera de la institución; si se cierra la guardería, controlar el acceso a otras guarderías para no extender la infección a nuevos colectivos; prohibir en las guarderías los fómites que imiten comidas, cubiertos y teléfonos; revisar nuestro sistema de vigilancia y participar en Strep-EURO; y finalmente, dada la presión para actuar rápidamente, desarrollar un protocolo para el manejo de los casos que facilite el trabajo de los servicios de salud pública. 


\section{BIBLIOGRAFÍA}

1. Cockerill FR, III, MacDonald KL, Thompson RL, Roberson F, Kohner PC, Besser-Wiek J et al. An outbreak of invasive group A streptococcal disease associated with high carriage rates of the invasive clone among school-aged children. JAMA 1997; 277: 38-43.

2. Factor SH, Levine OS, Schartz B, Harrison LH, Farley MM, McGeer A et al. Invasive Group A Streptococcal disease: risk factors for adults. Emerg Infect Dis 2003, 9: 970-7.

3. Stevens DL. Streptococcal toxic-shock syndrome: spectrum of disease, pathogenesis, and new concepts in treatment. Emerg Infect Dis 1995, 3: 6978.

4. Lamagni T, Efstratiou A, Vuopio-Varkila J, Jasir A, Schalen C. The epidemiology of severe Streptococcus pyogenes associated disease in Europe. Euro Surveill 2005; 10: 179-84.

5. Robinson KA, Rothrock G, Phan Q, Sayler B, Stefonek K, Beneden CV et al. Risk of severe Group A streptococcal disease among patients' household contacts. Emerg Infect Dis 2003, 9: 443-7.

6. Sierra JM, Sanchez F, Castro P, Salvado M, de la RG, Libois A et al. Group A streptococcal infections in injection drug users in Barcelona, Spain: epidemiologic, clinical, and microbiologic analysis of 3 clusters of cases from 2000 to 2003. Medicine (Baltimore) 2006; 85:139-46.

7. The Prevention of Invasive Group A streptococcal Infections Workshop Participants. Prevention of invasive group A streptococcal disease among household contacts of case patients and among postpartum and postsurgical patients: recommendations from the Centers for Disease Control and Prevention. Clin Infect Dis 2002; 35(8): 950-9.

8. Huang YC, Hseh PR, Lin TY, Yan DC, and Sia SH. A family cluster of streptococcal toxic shock syndrome in children: clinical implication and epidemiological investigation. Pediatrics 2001; 107: 1181-3.

9. Roy S, Kaplan EL, Rodriguez B, Schreiber JR, Salata RA, Palavecino E et al. A family cluster of five cases of group A streptococcal pneumonia. Pediatrics 2003; 112 (1 Pt 1):e61-e65.

10. Centers for Disease Control and Prevention. Outbreak of group A streptococcal pneumonia among Marine Corps recruits--California, November 1December 20, 2002. MMWR 2003; 52:106-9.
11. Smith A, Li A, Tolomeo O, Tyrrell GJ, Jamieson F and Fisman D. Mass antibiotic treatment for group A Streptococcus outbreaks in two long-term care facilities. Emerg Infect Dis 2003; 9:1260-5.

12. Manning SE, Lee E, Bambino M, Ackelsberg J, Weiss D, Sathyakumar C et al. Invasive group A streptococcal infection in high school football players, New York City, 2003. Emerg Infect Dis 2005; 11:146-9.

13. Centers for Disease Control and Prevention. Outbreak of invasive group A Streptococcus associated with varicella in a childcare center -- Boston, Massachusetts, 1997. MMWR 1997; 46:944-8.

14. Vallalta-Morales.M, Salavert-Lleti.M, AteroMora.A, Mahiques-Santos.L, Solaz-Moreno.E, Pérez-Bellés. C. Síndrome del shock tóxico estreptocócico: experiencia de los últimos 10 años en un hospital terciario. Enferm Infecc Microbiol Clin 2005; 23:540-4.

15. Factor SH, Levine OS, Harrison LH, Farley MM, McGeer A, Skoff T et al. Risk factors for pediatric invasive group A streptococcal disease. Emerg Infect Dis 2005; 11:1062-6.

16. Hollm-DFelgado MG, Allard R and Pilon PA. Invasive Group A streptococcal infections, clinical manifestations and their predictors, Montreal, 19095-2001. Emerg Infect Dis 2005, 11: 77-82.

17. Brogan TV. Varicella Vaccine Decreases Hospitalization in Varicella-Related Group A Strep Infections. AAP grand rounds 2004; 11:50-1.

18. Public Health Agency of Canada. Guidelines for the prevention and control of invasive Group A streptococcal disease. CCDR 2006; 32S2: 1-26.

19. Lesko SM, O'Brien KL, Schwartz B, Vezina R, Mitchell AA. Invasive group A streptococcal infection and nonsteroidal antiinflammatory drug use among children with primary varicella. Pediatrics 2001; 107:1108-15.

20. Roos K, Ino L and Hola SE. Estreptococos betahemolíticos del grupo A en un gato como posible fuente de amigdalitis de repetición en una familia. The Lancet (ed. Esp.) 1989, 3: 85

21. Smith TD, Wilkinson V and Kaplan EL. Group A streptococcal-associated upper respiratory tract infections in a day-care center. Pediatrics 1989, 83: $380-4$

22. Martin JM, Green M, Barbadora KA, Wald ER. Group A streptococci among school-aged chil- 
dren: clinical characteristics and the carrier state. Pediatrics 2004; 114:1212-9.

23. Heiter BJ, Bourbeau PP. Comparison of the GenProbe Group A Streptococcus Direct Test with culture and a rapid streptococcal antigen detection assay for diagnosis of streptococcal pharyngitis. J Clin Microbiol 1993; 31:2070-3.

24. Center for Disease Control and Prevention. Nosocomial Group A streptococcal infections associated with asymptomatic health-care workersMaryland and California, 1997. MMWR 1999; 48:163-6

25. Tyrrell GJ, Lovgren M, Kress B, Grimsrud K. Invasive group A streptococcal disease in Alberta, Canada (2000 to 2002). J Clin Microbiol 2005; 43:1678-83.

26. O'Brien KL, Beall B, Barrett NL, Cieslak PR, Reingold A, Farley MM et al. Epidemiology of invasive group A Streptococcus disease in the United States, 1995-1999. Clin Infect Dis 2002; 35:268-76.

27. Voyich JM, Sturdevant DE, Braughton KR, Kobayashi SD, Lei B, Virtaneva K et al. Genome-wide protective response used by group A Streptococcus to evade destruction by human polymorphonuclear leukocytes. Proc Natl Acad Sci USA 2003; 100:1996-2001.

28. Beres SB, Richter EW, Nagiec MJ, Sumby P, Porcella SF, DeLeo FR et al. Molecular genetic anatomy of inter- and intraserotype variation in the human bacterial pathogen group A Streptococcus. Proc Natl Acad Sci USA 2006; 103:7059-64.

29. Smith A, Lamagni TL, Oliver I, Efstratiou A, George RC, Stuart JM. Invasive group A streptococcal disease: should close contacts routinely receive antibiotic prophylaxis?. Lancet Infect Dis 2005; 5:494-500.

30. Prevention of invasive group A streptococcal disease among household contacts of case-patients: is prophylaxis warranted? The Working Group on Prevention of Invasive Group A streptococcal Infections. JAMA 1998; 279:1206-10.

31. Interim UK guidelines for management of close community contacts of invasive group A streptococcal disease. Commun Dis Public Health 2004; 7:354-61. 
